\title{
Stability of persistent currents in open dissipative quantum fluids
}

\author{
Guangyao Li, ${ }^{1}$ Michael D. Fraser, ${ }^{2}$ Alexander Yakimenko, ${ }^{3}$ and Elena A. Ostrovskaya ${ }^{1}$ \\ ${ }^{1}$ Nonlinear Physics Centre, Research School of Physics and Engineering, The Australian National University, Canberra ACT 2601, Australia \\ ${ }^{2}$ Quantum Functional System Research Group, RIKEN Center for Emergent Matter Science, 2-1 Hirosawa, Wako-shi, \\ Saitama 351-0198, Japan, \\ ${ }^{3}$ Department of Physics, Taras Shevchenko National University, Kyiv 01601, Ukraine \\ (Received 8 August 2014; revised manuscript received 18 March 2015; published 29 May 2015)
}

\begin{abstract}
The phenomenon of stable persistent currents is central to the studies of superfluidity in a range of physical systems. While most of the previous theoretical studies of superfluid flows in annular geometries concentrated on conservative systems, here we extend the dynamical stability analysis of persistent currents to open dissipative exciton-polariton superfluids. By considering an exciton-polariton condensate in an optically induced annular trap, we determine dynamical stability conditions for an initially imposed flow with a nonzero orbital angular momentum. We show, theoretically and numerically, that the system can sustain metastable persistent currents in a large parameter region, and describe scenarios of the supercurrent decay due to the dynamical instability.
\end{abstract}

DOI: 10.1103/PhysRevB.91.184518

PACS number(s): 67.85.De, 03.75.Kk, 05.60.Gg, 71.36.+c

\section{INTRODUCTION}

Superfluidity, which is an ability of a fluid to flow without friction, has been studied in various systems including the superfluid helium [1-3], superconductivity [4], Bose-Einstein condensate (BEC) of dilute atomic gases [5], and, more recently, exciton-polariton BECs in semiconductor microcavities [6-9].

One of the most important predictions of quantum hydrodynamics is the formation of persistent currents of a superfulid confined in an annular trap with an initially imposed rotation. Apart from the fundamental interest in this problem, ultrasensitive interferometric devices based on persistent currents have been suggested [10-12]. The ability to use the Laguerre-Gaussian (LG) mode of an optical laser to trap atomic BECs and transfer orbital angular momentum (OAM) from photons to atoms $[11,13,14]$ fuelled intensive studies of persistent currents in atomic condensates. Stability analysis [15-19] confirmed that persistent currents in ultracold atomic gases are metastable states with lifetimes limited only by the longevity of the BEC [14]. Although these theoretical studies agree with experiments, their scope is limited to conservative systems at thermal equilibrium.

Applicability of the existing theories to the novel quantum fluids formed by exciton-polariton condensates is questionable. Polaritons are quasiparticles arising from strong coupling between photons confined in a microcavity and excitions in a quantum well $[6,8]$. The polariton condensates can be generated either by coherent (resonant) or incoherent (off-resonant) optical pumping schemes. While the former leads to a condensate which is driven directly by the pumping laser [20], the later relies on nonradiative energy relaxation processes and stimulated scattering into the lowest energy state, which leads to a spontaneously established coherence [21]. Regardless of the excitation scheme, and in contrast to ultracold atomic gasses, an exciton-polariton $\mathrm{BEC}$ is an intrinsically nonequilibrium system because of the pumping and radiative decay of polaritons. With the growing experimental effort on creating persistent flows of open dissipative condensates [22-27], the urgent open question is how the intrinsic gain and loss would affect their stability.
In this work, we address this problem by constructing a comprehensive theory of polariton condensates with nonzero OAM supported by an optically induced annular confinement. We focus on an incoherent, off-resonant pumping scheme, which offers the possibility to engineer a trapping potential landscape by shaping the optical pump beam [25,28-31] and ensures that the condensate's phase evolution is not driven by the pump. We predict that persistent currents of polaritons with sufficiently high angular momenta are always prone to oscillatory dynamical instabilities. However, in sizable regions of the parameter space, the quantized circulation can persist almost indefinitely.

\section{THE MODEL}

The off-resonantly pumped polariton condensate can be described by the mean-field dissipative Gross-Pitaevskii (GP) equation for the macroscopic wave function $\psi$, coupled to the rate equation for the density of the excitonic reservoir $n_{R}$ [32]:

$$
\begin{aligned}
i \hbar \frac{\partial \psi}{\partial t} & =\left[-\frac{\hbar^{2}}{2 M} \nabla^{2}+g_{c}|\psi|^{2}+g_{R} n_{R}+\frac{i \hbar}{2}\left(R n_{R}-\gamma_{c}\right)\right] \psi, \\
\frac{\partial n_{R}}{\partial t} & =P-\left(\gamma_{R}+R|\psi|^{2}\right) n_{R},
\end{aligned}
$$

where $P(\mathbf{r}, t)$ is the pumping rate, $g_{c}$ and $g_{R}$ characterize the polariton-polariton and polariton-exciton interactions, respectively. The relaxation rates $\gamma_{c}$ and $\gamma_{R}$ quantify the finite lifetime of condensed polaritons and the reservoir, respectively. The stimulated scattering rate, $R$, controls the growth of the condensate density.

The mean-field model (1) is phenomenological, and cannot properly describe the condensation dynamics because it is not frequency selective [33]. Its limitations and connections with other models will be discussed in Sec. VI. Nevertheless, here we focus on the problem of stability of a formed condensate with a certain angular momentum, and therefore the assumption of a macroscopic polariton occupation in a condensed state is appropriate. Furthermore, the model conforms to the assumption that the far-off-resonant, incoherent optical $\mathrm{cw}$ excitation prohibits transfer of the pump phase to the 
condensate due to the accompanying phonon and exciton relaxation processes.

In what follows, we will consider the dimensionless form of Eq. (1) obtained by introducing the characteristic time $T=$ $\gamma_{c}^{-1}$, length $L=\sqrt{\hbar /\left(M \gamma_{c}\right)}$, and energy $E=\hbar \gamma_{c}$ scales [34].

Optical trapping techniques [22-24,28-31] rely on effective trapping potentials for polaritons created due to polariton flows and interaction with the reservoir. In the spirit of this approach, the annular condensate can be supported by the LG pump beam, with the LG mode intensity defining the spatial distribution of the condensed state. The OAM carried by the LG beam will not be transferred to the condensate because it is replenished from the incoherent excitonic reservoir, which "scrambles" the phase. Within the homogeneous approximation, the threshold of pumping rate to build up a nonzero condensate density is $P_{t h}=\gamma_{R} \gamma_{c} / R$ [32].

\section{STEADY CURRENTS}

Equations (1) with the radially symmetric pump, $P(r)$, admit steady states of the form: $\psi=\Psi(r, \theta) \exp (i m \theta) \exp (-i \mu t)$, where $\mu$ is the energy (chemical potential) of the steady state, $(r, \theta)$ are the polar coordinates, and $m$ is the phase winding number [35] (topological charge of a vortex) with the ground state corresponding to $m=0$. Such steady states can be found by solving (1) numerically, with the initially imposed vorticity $\psi(0)=A r^{|m|} \exp (i m \theta)$, and some examples for $m=0$ and $m \neq 0$ are presented in Fig. 1. Remarkably, the radial profiles of the condensate show an extremely weak dependence on $m$ [Fig. 1 (c)]. In experiment, the initial vorticity can be imprinted, e.g., by a pulsed resonant transfer of the orbital angular momentum onto an established $m=0$ state [36].

Within the pump area, quantized superfluid flows are supported purely by the balance of gain and loss, and therefore

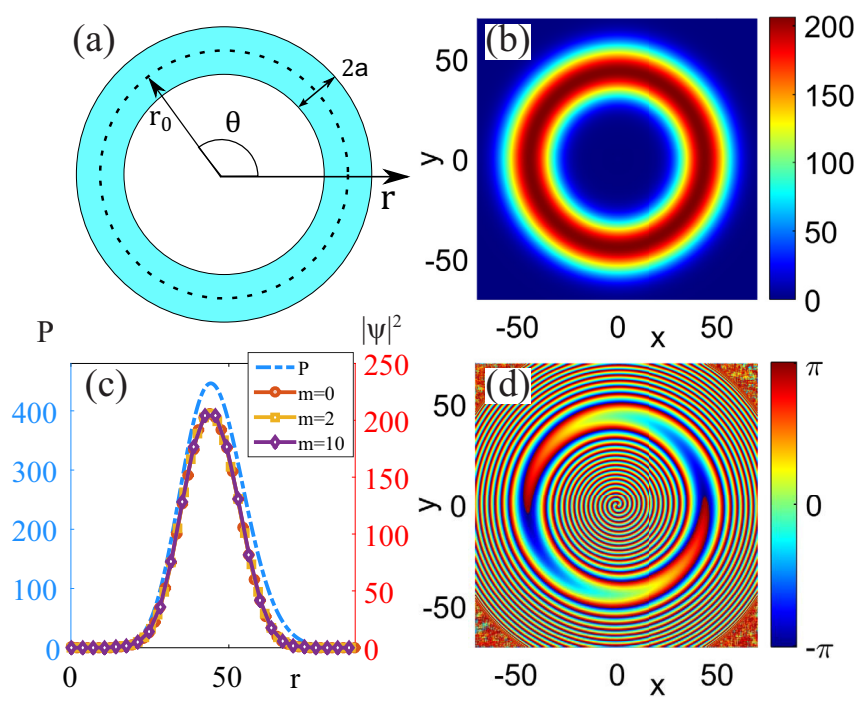

FIG. 1. (Color online) (a) Schematics of the radial structure of a LG pump beam; steady-state (b) density and (d) phase of the condensate for $m=2$; (c) radial profiles of the condensate steady states with $m=0,2,10$ supported by the $\mathrm{LG}_{50}$ mode with the pump rate profile $P(r)$ (dashed line). resemble dissipative vortex solitons in a focusing optical media [37]. At the same time, the steady state maintains inward and outward polariton flows outside the pump area, which creates an effective trapping potential in the radial direction [38,39].

In numerical simulations, the steady states are characterized by the conserved real part of the full energy functional $E_{0}$ and angular momentum $L_{z}$ :

$$
\begin{gathered}
E_{0}=\int\left[\frac{1}{2}|\nabla \psi|^{2}+g_{R} n_{R}|\psi|^{2}+\frac{g_{c}}{2}|\psi|^{4}\right] r d r d \theta, \\
L_{z}=\frac{i}{2} \int\left(\psi \frac{\partial \psi^{*}}{\partial \theta}-\psi^{*} \frac{\partial \psi}{\partial \theta}\right) r d r d \theta .
\end{gathered}
$$

For a steady state with azimuthally homogeneous density, the normalised angular momentum is equal to the topological charge of the vortex: $L_{z} / \int|\psi|^{2} r d r d \theta=m$.

When the radius of the LG beam is much larger than the width of the annulus, i.e., $r_{0} \gg a$, one can separate the radial and azimuthal dependence of the condensate wave function $[15,18,40,41]$ and derive a one-dimensional model, which was shown to agree with its higher-dimensional counterparts in the conservative case [16]. To this end, we set $\psi(r, \theta)=\Phi_{0}(r) \psi(\theta, t)$, where $r \in\left[r_{0}-a, r_{0}+a\right]$ and $\Phi_{0}(r)$ is assumed to take a constant value over the width of the ring, $2 a$. Substituting this ansatz into our model, and integrating out the radial dependence, we arrive at the reduced 1D model:

$$
\begin{aligned}
i \frac{\partial \psi(\theta, t)}{\partial t}= & \left\{-\frac{1}{2 r_{0}^{2}} \frac{\partial^{2}}{\partial \theta^{2}}+g_{c} n_{c}^{0}|\psi(\theta, t)|^{2}+g_{R} n_{R}(\theta, t)\right. \\
& \left.+\frac{i}{2}\left[R n_{R}(\theta, t)-\gamma_{c}\right]\right\} \psi(\theta, t), \\
\frac{\partial n_{R}(\theta, t)}{\partial t}= & P(\theta)-\left(\gamma_{R}+R n_{c}^{0}|\psi(\theta, t)|^{2}\right) n_{R}(\theta, t),
\end{aligned}
$$

where, assuming our normalization, $\gamma_{c}=1$.

For a steady state, which is homogeneous in the radial direction, gain balances loss: $R n_{R}^{0}=\gamma_{c}$, were $n_{R}^{0}$ is the steady-state reservoir density. The chemical potential of the stationary condensate with the azimuthal wave function $\psi(\theta, t)=\psi_{\theta}^{0}=\exp (i m \theta) \exp (-i \mu t)$ is given by $\mu=$ $m^{2} /\left(2 r_{0}^{2}\right)+g_{c} n_{c}^{0}+g_{R} n_{R}^{0}$, where $n_{c}^{0}=\Phi_{0}^{2}=\gamma_{R}(\bar{P}-1) / R$ is the condensate density, and $\bar{P}=P / P_{t h}$.

\section{STABILITY ANALYSIS}

The stability of the steady states with nonzero angular momentum can be analyzed following the standard Bogoliubovde Gennes (BdG) approach [5], by calculating the spectrum of the elementary excitations of the condensate and the reservoir in our reduced one-dimensional model: $\psi(\theta, t)=\psi_{\theta}^{0}(1+\delta \psi)$, and $n_{R}(t)=n_{R}^{0}+\delta n_{R}$. The excitations of the steady state and its reservoir are introduced in the form $[32,42]$

$$
\begin{aligned}
\delta \psi & =u_{0} e^{i k \theta-i \omega t}+v_{0}^{*} e^{-i k \theta+i \omega^{*} t}, \\
\delta n & =w_{0} e^{i k \theta-i \omega t}+w_{0}^{*} e^{-i k \theta+i \omega^{*} t} .
\end{aligned}
$$

Inserting $\psi(\theta)$ and $n_{R}$ into Eq. (4), and keeping only linear terms, we obtain the BdG equations: $\mathcal{L}_{m}(k) \mathcal{U}=\omega \mathcal{U}$, where 

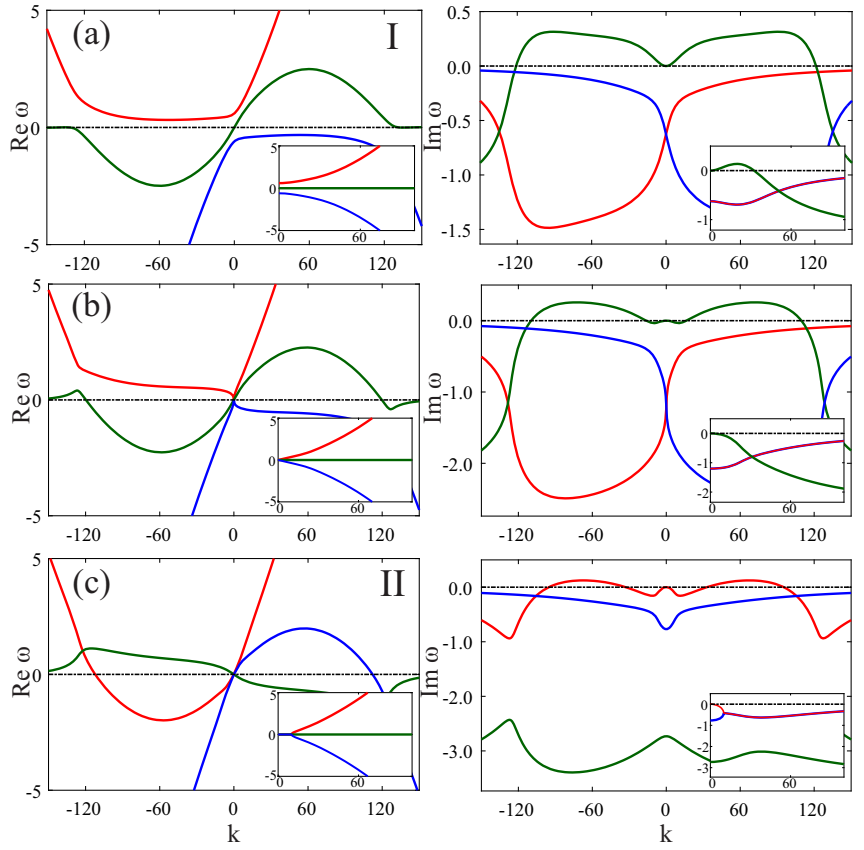

FIG. 2. (Color online) Dispersion curves $\operatorname{Re}[\omega(k)]=\Omega(k)$ and $\operatorname{Im}[\omega(k)]=\Gamma(k)$ for fixed $\bar{P}=2.5, m=60$, and (a) $P_{0}<\gamma_{c} / \gamma_{R}$, (b) $P_{0}=\gamma_{c} / \gamma_{R}$, (c) $P_{0}>\gamma_{c} / \gamma_{R}$. Insets show the corresponding dispersion for $m=0$.

$\mathcal{U}=\left(u_{0}, v_{0}, w_{0}\right)^{T}$, and

$\mathcal{L}_{m}(k)=\left[\begin{array}{ccc}\left(k_{+}+g_{c} n_{c}^{0}\right) & g_{c} n_{c}^{0} & \left(g_{R}+\frac{i}{2} R\right) \\ -g_{c} n_{c}^{0} & \left(k_{-}-g_{c} n_{c}^{0}\right) & -\left(g_{R}-\frac{i}{2} R\right) \\ -i \gamma_{c} n_{c}^{0} & -i \gamma_{c} n_{c}^{0} & -i\left(\gamma_{R}+R n_{c}^{0}\right)\end{array}\right]$.

Here, $k_{ \pm}= \pm(1 / 2)\left(\bar{k}^{2} \pm 2 \bar{k} \bar{m}\right)$ and $\{\bar{k}, \bar{m}\}=\left\{k / r_{0}, m / r_{0}\right\}$.

The spectrum of elementary excitations for $m=0$ is well known $[8,32,43-45]$. For $m \neq 0$, the dispersion relation given by the BdG equations is

$$
\begin{aligned}
& \omega^{3}-\omega^{2}\left(2 \bar{k} \bar{m}-i \bar{\gamma}_{R}\right) \\
& \quad-\omega\left(\omega_{B}^{2}-\bar{k}^{2} \bar{m}^{2}+R \bar{\gamma}_{c}-2 i \bar{\gamma}_{R} \bar{k} \bar{m}\right)-f(\bar{k}, \bar{m})=0,
\end{aligned}
$$

where $f(\bar{k}, \bar{m})=\bar{\gamma}_{c}\left(i g_{R} \bar{k}^{2}+R \bar{k} \bar{m}\right)+i \bar{\gamma}_{R}\left(\omega_{B}^{2}-\bar{k}^{2} \bar{m}^{2}\right), \omega_{B}^{2}=$ $\bar{k}^{4} / 4+g_{c} \Phi_{0}^{2} \bar{k}^{2}$ is the standard Bogoliubov dispersion, and we introduced the shorthand notations: $\bar{\gamma}_{c}=\gamma_{c} \Phi_{0}^{2}=P_{t h}(\bar{P}-1)$ and $\bar{\gamma}_{R}=\gamma_{R}+R \Phi_{0}^{2}=\gamma_{R} \bar{P}$.

At $k=0$, the real part of the excitation frequency $\omega(0)=$ $\Omega(0)+i \Gamma(0)$ is found as $\Omega^{2}(0)=R \bar{\gamma}_{c}-\bar{\gamma}_{R}^{2} / 4$. Consequently, it turns to zero for a critical pumping power $P_{0} \equiv \bar{P}^{2} /[4(\bar{P}-$ $1)]=\gamma_{c} / \gamma_{R}$, and the spectrum near $k=0$ resembles the Bogoliubov dispersion. For $P_{0}<\gamma_{c} / \gamma_{R}$, the Goldstone mode $(\omega=0)$ at $k=0$ is separated from the nonzero mode by a gap of the size $\Omega^{2}(0)>0$ [46]. For $P_{0}>\gamma_{c} / \gamma_{R}, \Omega^{2}(0)<0$ and the excitations exhibit a diffusive behavior near $k=0[32,43,47]$. The gapped and diffusive characters of the excitation spectra can be linked, respectively, to the underdamped and overdamped oscillations of the reservoir discussed in Ref. [46]. Figure 2 shows typical dispersion curves for the $m \neq 0$ in the gapped (a) and diffusive (c) regimes and the marginal case $P_{0}=\gamma_{c} / \gamma_{R}$ [Fig. 2(b)]. In what follows, without the loss of generality, we assume the condition $m>0$.
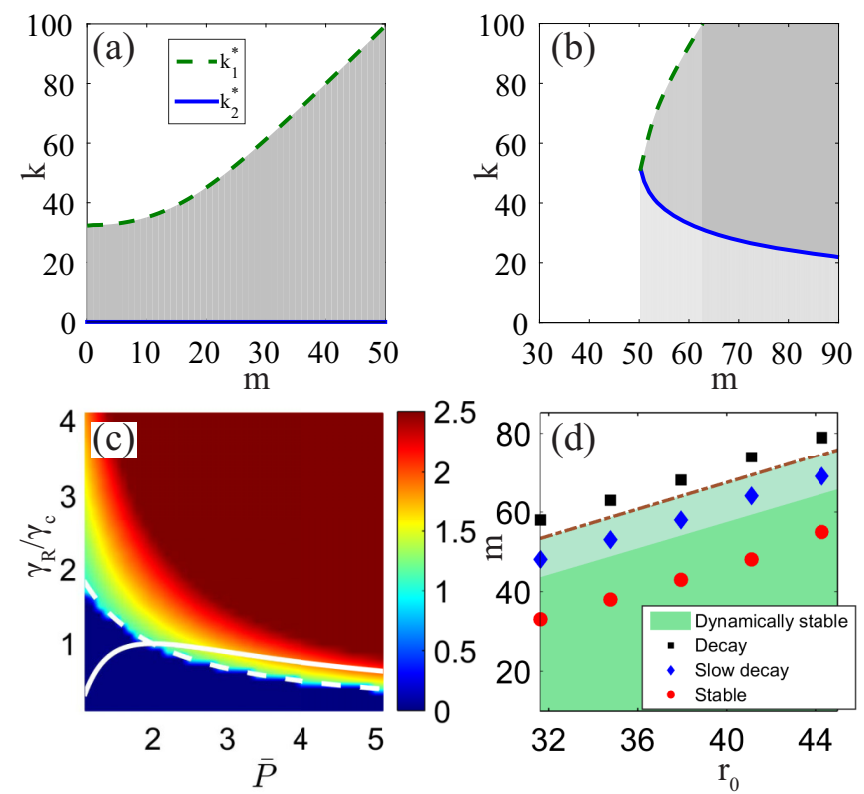

FIG. 3. (Color online) Critical values of $k_{1}^{*}$ and $k_{2}^{*}$ defining the modulational instability domains (shaded) in the regimes (a) $I$ and (b) II corresponding to Figs. 2(a) and 2(c), respectively. (c) Phase diagram of $m_{\mathrm{ds}} / r_{0}$. Dashed line, $\bar{P}=\left(g_{R} / g_{c}\right)\left(\gamma_{c} / \gamma_{R}\right)$, separates the MI regimes $I$ and $I I$. Solid line is given by $P_{0}=\gamma_{c} / \gamma_{R}$ (see text); (d) stability domains in the $m$ vs $r_{0}$ plane. Dots correspond to the $2 \mathrm{D}$ numerical simulations, the boundary (dashed), $m_{\mathrm{ds}}$, of the dynamically stable (shaded) region is given by the $1 \mathrm{D}$ theory.

When the imaginary part of the excitation frequency becomes positive, $\Gamma(k)>0$, for an interval $k_{2}^{*}<k<k_{1}^{*}$, the corresponding steady state experiences modulational (dynamical) instability (MI). Although the rotationally symmetry of the flow is preserved [48], its instability stems from the open dissipative nature of the polariton system [32]. As seen from Fig. 2, for $m \neq 0$, the corresponding real part of the excitation frequency is always nonzero, $\Omega(k) \neq 0$, which indicates the oscillatory nature of the instability. The polariton current exhibits MI only above certain critical OAM, $m>m_{\mathrm{ds}}$, which is defined by $\Gamma(k)$ crossing into the positive half-plane, at which point $k_{1}^{*}=k_{2}^{*} \neq 0$. Two regimes of instability can be identified.

Regime I corresponds to $m_{\mathrm{ds}}=0$ and is defined by the condition $\bar{P}<\left(g_{R} / g_{c}\right)\left(\gamma_{c} / \gamma_{R}\right)$ [45]. In this regime, the ground state $m=0$ is modulationally unstable, and $k_{2}^{*}=0$, as shown in the inset on the right panel of Figs. 2(a) and 3(a). The real part of the corresponding excitation frequency is zero, $\Omega(k)=0$, so that perturbations of the $m=0$ state grow exponentially and lead to fragmentation of the azimuthally homogeneous steady state. In this parameter range, due to the saturable nature of gain in this system, the effective nonlinearity becomes attractive for low condensate densities [45]. As seen from Fig. 3(c) (below dashed line), this regime mostly overlaps with the gapped domain of the excitation spectra (below the solid line). Physically, this behavior appears to be most relevant near the condensation threshold, $\bar{P} \sim 1$ due to the long lifetimes of the reservoir compared to condensate polaritons $\gamma_{R} / \gamma_{c}<1$.

Regime II corresponds to $m_{\mathrm{ds}}>0$ and $\bar{P}>\left(g_{R} / g_{c}\right)\left(\gamma_{c} / \gamma_{R}\right)$ [Fig. 3(c), above dashed line]. In this regime, the ground 
state $m=0$ is dynamically stable, and the 1D theory predicts dynamical stability of the flow against azimuthal density modulations up to reasonably high values of $m_{\mathrm{ds}}$ [Figs. 3(b) and 3(c)].

\section{DYNAMICS OF THE PERSISTENT CURRENTS}

Numerical simulations of the full 2D model (1) with a weak, incoherent perturbation applied to the steady current, show remarkable agreement with the predictions of the 1D stability theory. Indeed, in the regime $I I$, for $m>m_{\mathrm{ds}}$, the initial stage of the instability development manifests in oscillating and rapidly growing density perturbations [Fig. 4(d)], whereby the condensate fragments [Fig. 4(b)]. Fluctuations around the steady state grow without the formation of surface modes $[49,50]$, confirming the validity of our $1 D$ approximation. During the long-term, nonlinear stage of instability development, the azimuthal flow "heals" [Fig. 4(c)], and the system attains a new, dynamically stable steady state [Fig. 4(a)]. Figure 4 shows a typical scenario of the oscillatory instability development causing the system to enter a steady state with a reduced OAM and energy.

In contrast, in the regime $I$, where $m_{\mathrm{ds}}=0$, once the dynamical instability of the persistent current is triggered, the steady flow never recovers [Fig. 5]. The rate of instabilitytriggered decay depends on the maximum instability growth rate, $\max (\Gamma)$, which accounts for the broad transition region from dynamically unstable to stable regime depicted in Fig. 3(d).

In the annular geometry, steady-state transitions can be observed only in the MI domain, $m>m_{\mathrm{ds}}$. Indeed, in this parameter domain, the modulationally unstable current undergoes fragmentation and experiences phase slips, thus enabling the condensate to reduce its OAM (see Fig. 4). Outside the MI domain, the exponential decay of excitations suppresses the development of instability, and the vortex flow remains dynamically stable. Indeed, for $m \ll m_{\mathrm{ds}}$ [red dots in
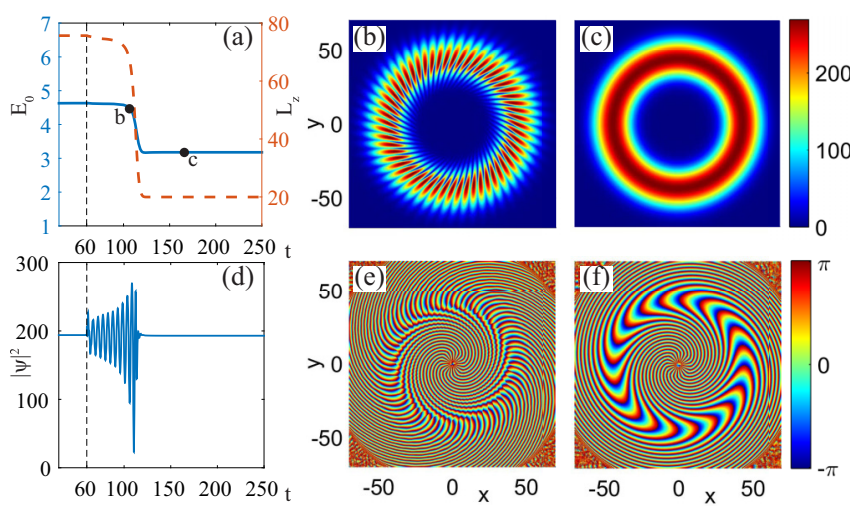

FIG. 4. (Color online) Evolution of a dynamically (modulationally) unstable state with $m>m_{\mathrm{ds}}$ in the regime $I I$. (a) Energy and (normalised) angular momentum evolution during steady state switching triggered by the oscillatory instability; (b) and (c) density and (e) and (f) phase distribution at the (b) and (e) intermediate and (c) and (f) final stages of evolution. (d) Peak density evolution at an arbitrary point. Dashed line in (a) and (d) indicates introduction of a weak pulsed perturbation.
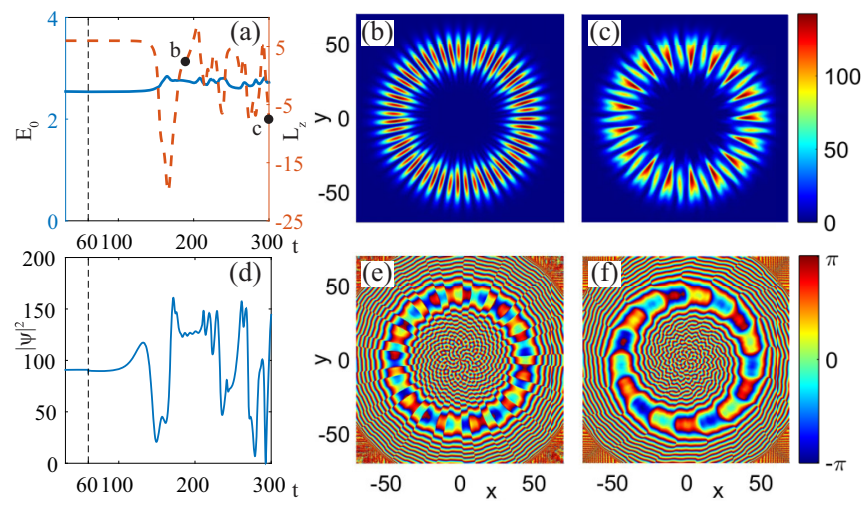

FIG. 5. (Color online) Evolution of a dynamically (modulationally) unstable state with $m>0$ in the regime $I$. (a) Energy (2) and (normalised) angular momentum (3) evolution during the decay of the $m=7$ current triggered by oscillatory instability; (b) and (c) density and (e) and (f) phase distribution at the (b) and (e) intermediate and (c) and (f) final stages of evolution. (d) Peak density evolution at an arbitrary point. Dashed line in (a) and (d) indicates the introduction of a weak pulsed perturbation.

Fig. 3(d)], we do not observe decay of the persistent flow in our full 2D numerical simulations of the model.

The above reasoning is consistent with the previous numerical study of a single charge polariton vortex in a wide annulus geometry [51]. The loss of the spatial coherence of the condensate shown to be associated with the decay of the circulation [51], is a typical signature of the MI triggered by fluctuations [52].

\section{CONCLUSIONS}

We have analyzed the dynamical (modulational) instability of the persistent currents in dissipative polariton condensates confined by all-optical annular traps and predicted that a current with an orbital angular momentum within the modulationally stable domain will persist. Above the critical values of the OAM, the flows suffer from oscillatory instabilities, which leads to either dynamical switching to a new metastable steady state with a lower OAM, or to fragmentation and destruction of the superfluid flow. Within the framework of our model, the transition between different steady states is triggered by the dynamical instability, whereas possible investigations of energetic instability $[19,56]$ should additionally account for energy relaxation processes in the exciton-polariton system.

Owing to the complexity of the open dissipative nature of polariton systems, there exist several different phenomenological models that feature complex pumping and decay mechanisms [44]. It is therefore instructive to discuss the relevance of our results to specific features of our model.

First, the influence of the reservoir on the condensate dynamics gives rise to the appearance of regime $I$, in which even the ground state with zero orbital angular momentum is modulationally unstable. As argued in Ref. [32], and supported by later studies [45,52,53], when the reservoir mode participates fully in the dynamics, the gain saturation (the so-called hole-burning effect) will take place and lead to the fragmentation of the condensate. This can be seen in 
Fig. 2(a) where the instability is indeed driven by the reservoir mode (green curves). When the $\gamma_{R} / \gamma_{c}$ ratio or the $\bar{P}$ value is increased, however, the reservoir has little influence on the condensate dynamics. It can be seen in Fig. 2(c) that the reservoir mode is more (dynamically) stable than the condensate ones. In this regime, the reservoir can be regarded as indifferent to small perturbations and can be treated as static. This regime roughly corresponds to the area above both the solid line and the dashed line in the phase diagram Fig. 3(c). Many models [42,44,54,55] adopted the static reservoir assumption. The stability analysis in the framework of these models corresponds to our regime II or to the boundary area [Fig. 3(c) solid curve], where the Bogoliubov-like dispersion of elementary excitation is obtained [44].

Secondly, the stability of the persistent current is dramatically influenced by the open dissipative nature of the polariton system. The transition to a lower OAM steady state in our model (Fig. 4), is driven by the dynamically unstable modes that enable condensate fragmentation. This result can also be obtained by using models that account for energy relaxation, such as that in Ref. [42], since the dispersion curves for the elementary excitations are similar to those found in our model.

In conclusion, our results predict measurable limits of the dynamical stability of the persistent currents, which would affect their observability and possible utilization in polaritonic devices. The possibility to create a polariton condensate in an optically induced annular trap has already been explored experimentally [22-24,27]. Provided that the coherent imprinting of the orbital angular momentum [36] can be realized for these systems, experimental tests of our predictions are feasible.

\section{ACKNOWLEDGMENTS}

This work was supported by the Australian Research Council (ARC) through the Discovery and Future Fellowship schemes and by ImPACT Program of Council for Science, Technology and Innovation (Cabinet Office, Government of Japan). G.L. acknowledges support of the China Scholarship Council (CSC).
[1] O. Avenel and E. Varoquaux, Phys. Rev. Lett. 55, 2704 (1985).

[2] E. Varoquaux, M. W. Meisel, and O. Avenel, Phys. Rev. Lett. 57, 2291 (1986).

[3] J. C. Davis, J. Steinhauer, K. Schwab, Y. M. Mukharsky, A. Amar, Y. Sasaki, and R. E. Packard, Phys. Rev. Lett. 69, 323 (1992).

[4] J. F. Annett, Superconductivity, Superfluids, and Condensates (Oxford University Press, NY, 2004).

[5] L. Pitaevskii and S. Stringari, Bose-Einstein Condensation (Oxford University Press, NY, 2003).

[6] H. Deng, H. Haug, and Y. Yamamoto, Rev. Mod. Phys. 82, 1489 (2010).

[7] A. Amo, J. Lefrère, S. Pigeon, C. Adrados, C. Ciuti, I. Carusotto, R. Houdré, E. Giacobino, and A. Bramati, Nat. Phys. 5, 805 (2009).

[8] I. Carusotto and C. Ciuti, Rev. Mod. Phys. 85, 299 (2013).

[9] T. Byrnes, N. Y. Kim, and Y. Yamamoto, Nat. Phys. 10, 803 (2014).

[10] G. Franchetti, N. G. Berloff, and J. J. Baumberg, arXiv:1210.1187.

[11] A. Ramanathan, K. C. Wright, S. R. Muniz, M. Zelan, W. T. Hill, C. J. Lobb, K. Helmerson, W. D. Phillips, and G. K. Campbell, Phys. Rev. Lett. 106, 130401 (2011).

[12] C. Ryu, P. W. Blackburn, A. A. Blinova, and M. G. Boshier, Phys. Rev. Lett. 111, 205301 (2013).

[13] C. Ryu, M. F. Andersen, P. Cladé, V. Natarajan, K. Helmerson, and W. D. Phillips, Phys. Rev. Lett. 99, 260401 (2007).

[14] S. Moulder, S. Beattie, R. P. Smith, N. Tammuz, and Z. Hadzibabic, Phys. Rev. A 86, 013629 (2012).

[15] J. Javanainen, S. M. Paik, and S. M. Yoo, Phys. Rev. A 58, 580 (1998).

[16] L. Salasnich, A. Parola, and L. Reatto, Phys. Rev. A 59, 2990 (1999).

[17] J. Javanainen and Y. Zheng, Phys. Rev. A 63, 063610 (2001).

[18] S. Bargi, F. Malet, G. M. Kavoulakis, and S. M. Reimann, Phys. Rev. A 82, 043631 (2010).
[19] M. Modugno, C. Tozzo, and F. Dalfovo, Phys. Rev. A 70, 043625 (2004).

[20] P. G. Savvidis, J. J. Baumberg, R. M. Stevenson, M. S. Skolnick, D. M. Whittaker, and J. S. Roberts, Phys. Rev. Lett. 84, 1547 (2000).

[21] R. Huang, F. Tassone, and Y. Yamamoto, Phys. Rev. B 61 , R7854(R) (2000).

[22] F. Manni, K. G. Lagoudakis, T. C. H. Liew, R. André, and B. Deveaud-Plédran, Phys. Rev. Lett. 107, 106401 (2011).

[23] A. Dreismann, P. Cristofolini, R. Balili, G. Christmann, F Pinsker, N. G. Berloff, Z. Hatzopoulos, P. G. Savvidis, and J. J. Baumberg, Proc. Natl. Acad. Sci. 111, 8770 (2014).

[24] G. Liu, D. W. Snoke, A. Daley, L. Pfeier, and K. West, Proc. Natl. Acad. Sci. 112, 2676 (2015).

[25] R. Dall, M. D. Fraser, A. S. Desyatnikov, G. Li, S. Brodbeck, M. Kamp, C. Schneider, S. Höfling, and E. A. Ostrovskaya, Phys. Rev. Lett. 113, 200404 (2014).

[26] V. G. Sala, D. D. Solnyshkov, I. Carusotto, T. Jacqmin, A. Lemaître, H. Terças, A. Nalitov, M. Abbarchi, E. Galopin, I. Sagnes, J. Bloch, G. Malpuech, and A. Amo, Phys. Rev. X 5, 011034 (2015).

[27] T. Boulier, H. Terças, D. D. Solnyshkov, Q. Glorieux, E. Giacobino, G. Malpuech, and A. Bramati, Sci. Rep. 5, 9230 (2015).

[28] G. Roumpos, W. H. Nitsche, S. Höfling, A. Forchel, and Y. Yamamoto, Phys. Rev. Lett. 104, 126403 (2010)

[29] G. Tosi, G. Christmann, N. G. Berloff, P. Tsotsis, T. Gao, Z. Hatzopoulos, P. G. Savvidis, and J. J. Baumberg, Nat.Phys. 8, 190 (2012).

[30] A. Askitopoulos, H. Ohadi, A. V. Kavokin, Z. Hatzopoulos, P. G. Savvidis, and P. G. Lagoudakis, Phys. Rev. B 88, 041308(R) (2013).

[31] P. Cristofolini, A. Dreismann, G. Christmann, G. Franchetti, N. G. Berloff, P. Tsotsis, Z. Hatzopoulos, P. G. Savvidis, and J. J. Baumberg, Phys. Rev. Lett. 110, 186403 (2013).

[32] M. Wouters and I. Carusotto, Phys. Rev. Lett. 99, 140402 (2007). 
[33] M. Wouters, T. C. H. Liew, and V. Savona, Phys. Rev. B 82, 245315 (2010).

[34] Throughout the paper we use physical values that are reasonably close to those measured for AlGaAs microcavity polaritons: $g_{c}=6 \times 10^{-3} \mathrm{meV} \mu \mathrm{m}^{2}, g_{R}=2 g_{c}, \gamma_{c}=0.33 \mathrm{ps}^{-1}$, and $R=$ $0.01 \mu \mathrm{m}^{2} \mathrm{ps}^{-1}$. The lower polariton effective mass entering our model is related to the mass of free electron as $M=10^{-4} m_{e}$.

[35] A. S. Desyatnikov, Yu. S. Kivshar, and L. Torner, Prog. Opt. 47, 291 (2005).

[36] D. Sanvitto, F. M. Marchetti, M. H. Szymańska, G. Tosi, M. Baudisch, F. P. Laussy, D. N. Krizhanovskii, M. S. Skolnick, L. Marrucci, A. Lemaître, J. Bloch, C. Tejedor, and L. Viña, Nat. Phys. 6, 527 (2010).

[37] O. V. Borovkova, V. E. Lobanov, Y. V. Kartashov, and L. Torner, Phys. Rev. A 85, 023814 (2012).

[38] E. A. Ostrovskaya, J. Abdullaev, A. S. Desyatnikov, M. D. Fraser, and Yu. S. Kivshar, Phys. Rev. A 86, 013636 (2012).

[39] L. Ge, A. Nersisyan, B. Oztop, and H. E. Túreci, arXiv:1311.4847.

[40] S. Schwartz, M. Cozzini, C. Menotti, I. Carusotto, P. Bouer, and S. Stringari, New J. Phys. 8, 162 (2006).

[41] S. Baharian and G. Baym, Phys. Rev. A. 87, 013619 (2013).

[42] M. Wouters and I. Carusotto, Phys. Rev. Lett. 105, 020602 (2010).
[43] M. H. Szymańska, J. Keeling, and P. B. Littlewood, Phys. Rev. Lett. 96, 230602 (2006).

[44] D. D. Solnyshkov, H. Tercas, K. Dini, and G. Malpuech, Phys. Rev. A 89, 033626 (2014).

[45] L. A. Smirnov, D. A. Smirnova, E. A. Ostrovskaya, and Yu. S. Kivshar, Phys. Rev. B 89, 235310 (2014).

[46] T. Byrnes, T. Horikiri, N. Ishida, M. Fraser, and Y. Yamamoto, Phys. Rev. B 85, 075130 (2012).

[47] M. Wouters and I. Carusotto, Phys. Rev. A 76, 043807 (2007).

[48] D. S. Rokhsar, Phys. Rev. Lett. 79, 2164 (1997).

[49] R. Dubessy, T. Liennard, P. Pedri, and H. Perrin, Phys. Rev. A 86, 011602(R) (2012).

[50] S. J. Woo and Y.-W. Son, Phys. Rev. A 86, 011604 (2012).

[51] M. Wouters and V. Savona, Phys. Rev. B 81, 054508 (2010).

[52] N. Bobrovska, E. A. Ostrovskaya, and M. Matuszewski, Phys. Rev. B 90, 205304 (2014).

[53] T. C. H. Liew, O. A. Egorov, M. Matuszewski, O. Kyriienko, X. Ma, and E. A. Ostrovskaya, Phys. Rev. B 91, 085413 (2015).

[54] J. Keeling and N. G. Berloff, Phys. Rev. Lett. 100, 250401 (2008).

[55] M. O. Borgh, J. Keeling, and N. G. Berloff, Phys. Rev. B 81, 235302 (2010).

[56] B. Wu and Q. Niu, New J. Phys. 5, 104 (2003). 